
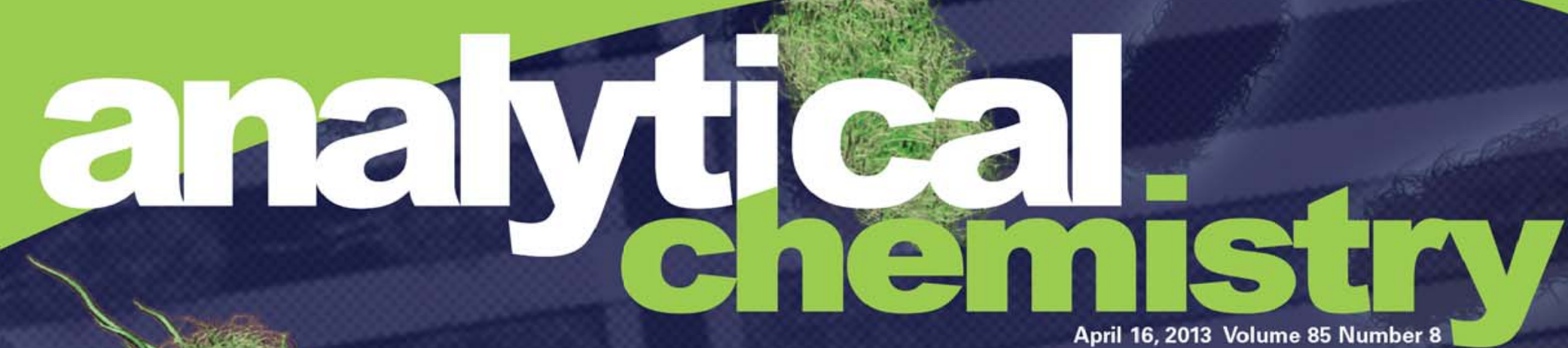

April 16, 2013 Volume 85 Number 8

Single Cell Antimicrobial Susceptibility Testing by Confined Microchannels and Electrokinetic Loading
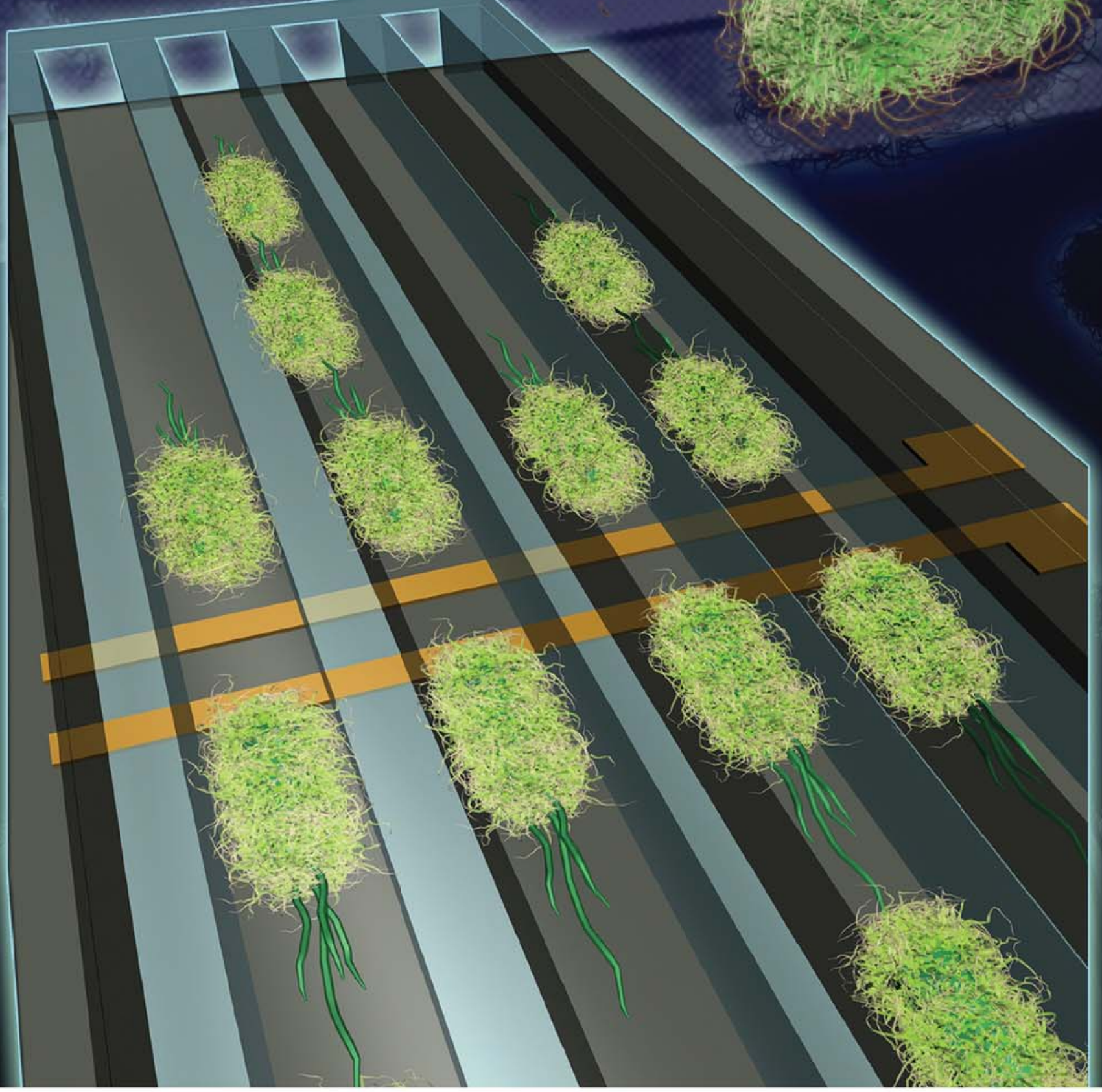


\title{
Single Cell Antimicrobial Susceptibility Testing by Confined Microchannels and Electrokinetic Loading
}

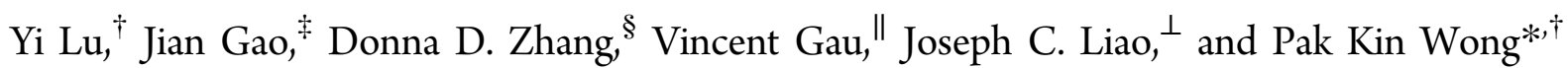 \\ ${ }^{\dagger}$ Department of Aerospace and Mechanical Engineering, The University of Arizona, Tucson, Arizona, 85721, United States \\ ${ }^{\ddagger}$ Department of Chemical Engineering, Shandong Polytechnic University, Jinan 250353, China \\ ${ }^{\S}$ Department of Pharmacology and Toxicology, The University of Arizona, Tucson, Arizona, 85721, United States \\ "GeneFluidics Inc., Irwindale, California, 91010, United States \\ ${ }^{\perp}$ Department of Urology, Stanford University, Palo Alto, California, 94304, United States
}

ABSTRACT: Multidrug-resistant pathogens are an emerging global health problem. In addition to the need of developing new antibiotics in the pipeline, the ability to rapidly determine the antibiotic resistance profiles of bacteria represents one of the most crucial steps toward the management of infectious diseases and the prevention of multidrug-resistant pathogens. Here, we report a single cell antimicrobial

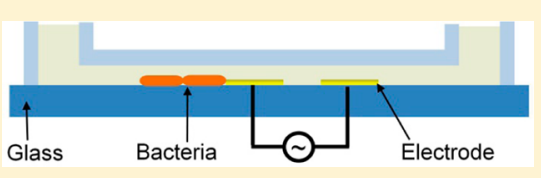
susceptibility testing (AST) approach for rapid determination of the antibiotic resistance of bacterial pathogens. By confining individual bacteria in gas permeable microchannels with dimensions comparable to a single bacterium, the antibiotic resistance of the bacteria can be monitored in real-time at the single cell level. To facilitate the dynamic loading of the bacteria into the confined microchannels for observation, AC electrokinetics is demonstrated for capturing bacteria to defined locations in highconductivity AST buffer. The electrokinetic technique achieves a loading efficiency of about $75 \%$ with a negligible effect on the bacterial growth rate. To optimize the protocol for single cell AST, the bacterial growth rate of individual bacteria under different antibiotic conditions has been determined systematically. The applicability of single cell AST is demonstrated by the rapid determination of the antimicrobial resistant profiles of uropathogenic clinical isolates in Mueller-Hinton media and in urine. The antibiotic resistance profiles of bacteria can be determined in less than $1 \mathrm{~h}$ compared to days in standard culture-based AST techniques.

$\mathrm{O}$ ne of the most common causes of health complications and mortality worldwide is infectious diseases caused by bacterial pathogens. ${ }^{1,2}$ Due to the widespread use of broadspectrum antibiotics, multidrug-resistant pathogens are rapidly emerging among bacterial pathogens and represent a major threat to global health. ${ }^{3-5}$ To allow for the proper clinical management of infectious diseases and determine the most effective treatment for patients, antimicrobial susceptibility testing (AST) is performed to evaluate the antibiotic resistance of the bacteria obtained in the patient samples such as urine, blood, sputum, or wound swabs. ${ }^{6,7}$ If the antibiotic resistance profile of the bacterial pathogen can be rapidly determined, physicians will be able to select the most appropriate treatment for the patients. However, standard culture-based AST approaches involve sample transportation to a centralized clinical microbiology laboratory and are not available in resource limited settings, such as physician offices, intensive care units, and rural clinics. In addition, the standard AST process from initial sample to result reporting typically requires $48-72 \mathrm{~h}$. As a result, the lack of rapid diagnostics has largely driven the overuse and misuse of broad-spectrum antibiotics. The widespread use of antibiotics consecutively accelerates the emergence of multidrug-resistant pathogens and further worsens the situation.

Rapid determination of the antibiotic resistance profile of the bacteria represents an essential step toward the proper management of infectious diseases caused by multidrug resistant pathogens. ${ }^{8-10}$ Several approaches have been developed for the determination of bacterial antibiotic resistance. For instance, molecular strategies can be applied to characterize the genotypic changes of bacteria when the resistance genes are known. ${ }^{11,12}$ Phenotypic or culture-based AST, which measures the growth of the bacteria in the presence of antibiotics, can also be performed to determine the most effective antibiotic for the bacterium. ${ }^{9,13}$ Phenotypic AST is often preferred over genotypic AST due to the diverse, evolving drug resistance mechanisms encountered. ${ }^{8,9}$ In fact, culturebased techniques, such as the Kirby-Bauer method (i.e., disk diffusion $)^{14}$ are particularly useful in resource-limited settings, although only qualitative results can be obtained. Other methods, such as measuring the change in impedance and $\mathrm{CO}_{2}$ release, have also been developed to quantitatively monitor the bacterial growth. ${ }^{15-20}$ However, these techniques require bulky, expensive supporting equipment, which could limit their use in resource-limited settings. Furthermore, most culture-based methods involve overnight bacterial culture and are labor intensive. Another major limitation of conventional AST techniques is the long assay time necessary to obtain statistically significant data.

Received: December 6, 2012

Accepted: February 14, 2013

Published: February 27, 2013 
Microfluidics has enabled new opportunities in developing novel approaches to address the current limitations in $\mathrm{AST}^{21-28}$ For instance, high surface-to-volume ratio, gaspermeable microfluidic channels can be applied to enhance system oxygenation and eliminate the requirement of bulky equipment for facilitating phenotypic AST in resource-limited settings. ${ }^{23}$ In particular, phenotypic AST has been demonstrated in a $250 \mu \mathrm{m}$ height microchannel in $2 \mathrm{~h}$. Multiphase microfluidics has also been reported to create stochastic confinement for culturing individual bacteria in nanoliter droplets. ${ }^{26}$ Using multiphase microfluidics with a fluorescence cell viability indicator to monitor the bacterial growth, antibiotic resistance of individual bacteria can be determined in $7.5 \mathrm{~h}$. Moreover, a dielectrophoresis (DEP)-based trapping technique with automated image analysis for single cell AST has been demonstrated, and growth curves can be determined in less than 5 h. ${ }^{24}$ On the other hand, physical confinement can be applied to track the growth of individual cells and has been demonstrated for studying bacterial persistence. ${ }^{29}$ Nevertheless, the feasibility of microfluidic confinement for phenotypic AST in resource-limited settings has not been demonstrated. To perform phenotypic AST with microfluidic confinement, the design and the operation conditions of the device, such as the geometry of the confinement channel and the sample loading procedure, should be optimized. The bacterial growth behavior, such as the distribution and variance, should also be characterized in order to determine the proper AST conditions.

In this study, we report a single cell AST approach with confined microchannels and electrokinetic loading. Escherichia coli (E. coli) clinical isolates from patients with urinary tract infection, which is the most common bacterial infection, are applied as the model pathogens in this study. By confining uropathogenic E. coli in gas permeable microchannels with dimensions compatible to a single bacterium, the growth of individual bacteria can be monitored at the single cell level. Furthermore, microelectrodes are integrated in the confinement channels to position the bacteria with DEP for facilitating the observation of bacterial growth in defined locations. DEP is highly effective in our configuration due to the close proximity between the electrode edge and the pathogens. The effect of the applied voltage is investigated to maximize the loading rate while minimizing the harmful electrothermal effect on bacterial growth. The kinetics of the bacterial growth rates among the bacterial population is also characterized to determine the proper assay time required for AST. The applicability of single cell AST is demonstrated by determining the antibiotic resistance profiles of uropathogenic clinical isolates in culture media and in urine samples in $1 \mathrm{~h}$.

\section{MATERIALS AND METHODS}

Microfluidic Chip. The microfluidic devices for single cell AST are shown in Figure 1a,b. The microchannels were fabricated by molding polydimethylsiloxane (PDMS) at $65{ }^{\circ} \mathrm{C}$ for $5 \mathrm{~h}$. The confinement channels tested had a width ranging from 0.5 to $10 \mu \mathrm{m}$. The device has one inlet and one outlet and consists of 68 parallel confinement channels. The number of channels can be increased to capture bacteria in samples with low concentrations. The master molds used to form the confinement channels were created via two separate methods. The masters for channels between 10 and $3 \mu \mathrm{m}$ were fabricated by photolithography, and the masters for channels from $2 \mu \mathrm{m}$ to $500 \mathrm{~nm}$ were created by electron beam lithography. The single cell AST device consisted of a set of parallel electrodes,
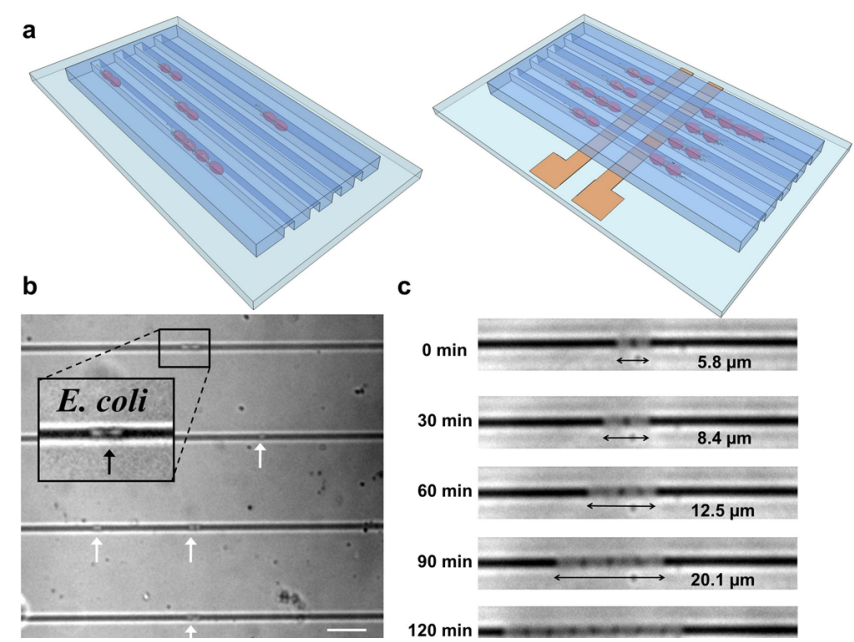

Figure 1. (a,b) Schematic diagrams of bacteria trapped in confined microchannels for single cell AST with and without electrokinetic loading. (c) Uropathogenic E. coli loaded at different locations in confined microchannels. White arrows indicate the position of the bacteria trapped in the channels. Scale bar is $10 \mu \mathrm{m}$. (d) Time lapse images of E. coli growing in a microchannel for $2 \mathrm{~h}$.

which were fabricated by sputtering $50 \mathrm{~nm}$ of titanium, $150 \mathrm{~nm}$ of gold, and $50 \mathrm{~nm}$ of titanium on a glass substrate and were patterned by a lift-off process. The length, width, and gap distance of each electrode was $7 \mathrm{~mm}, 100 \mu \mathrm{m}$, and $20 \mu \mathrm{m}$, respectively. The channel layer and electrode layer were bonded by air plasma treatment (PDC-001, Harrick Plasma). At the same time, the plasma treatment also sterilized the channels and increased the hydrophilicity of the channel surface for capillary sample loading. Passive loading with capillary force is applied to simplify the system requirement. Unlike loading with a syringe pump, capillary force avoids pressure buildup in the inlet due to the small dimension of the channels. Specifically, a $5 \mu \mathrm{L}$ drop of sample solution was placed at the inlet of the channel and capillary force automatically drove the sample solution into the channels. For high concentration samples (e.g., $10^{7}$ and $10^{8} \mathrm{cfu} / \mathrm{mL}$ ), bacterial loading can be finished in seconds. For samples with a low concentration (e.g., $10^{5}$ and $10^{6} \mathrm{cfu} / \mathrm{mL}$ ), the bacterial loading time can be increased to $5 \mathrm{~min}$ for capturing a sufficient number of bacteria for statistical analysis. The bacteria were then physically trapped in the confined channels and can be monitored continuously.

Experimental Setup. In the experiment, the single cell AST device was loaded onto a digital inverted epi-fluorescence microscope (DMI 4000B, Leica Microsystems, Wetzlar, Germany). All experiments were performed using a custommade microscope heating stage and incubator maintained at 37 ${ }^{\circ} \mathrm{C}$. In order to allow for optical inspection via bright field microscopy, the microscope stage and the incubator were directly mounted on the microscope. The morphology and density of the bacteria were captured by a CCD camera (Cooke DMI4000B SensiCAM QE). The images and videos were processed by NIH ImageJ software. The AC potential for electrokinetic loading is supplied using a function generator (Hewlett-Packard, 33120A). The AC potential is removed after bacteria loading.

Pathogens and Physiological Samples. We chose two uropathogenic E. coli clinical isolates (EC137 and EC132) in this study. Both of the isolates were obtained from the Veterans Affairs Palo Alto Health Care System (VAPAHCS). The 
uropathogenic clinical samples were isolated from patients with procedures approved by the Stanford University Institutional Review Board. The antimicrobial resistance profiles for EC132 and EC137 were previously determined by the clinical microbiology laboratory at VAPAHCS. EC137 and EC132 are both sensitive to antibiotics trimethoprim/sulfamethoxazole $\left(\mathrm{SXT}^{S}\right)$; however, EC137 is also sensitive to the antibiotics ampicillin and ciprofloxacin $\left(\mathrm{AMP}^{\mathrm{S}}, \mathrm{CIP}^{\mathrm{S}}\right) . \mathrm{EC} 132$ is resistant to ampicillin and ciprofloxacin $\left(\mathrm{AMP}^{\mathrm{R}}, \mathrm{CIP}^{\mathrm{R}}\right)$. The superscripts "S" and " $R$ " stand for sensitive and resistant, respectively.

Antibiotic Susceptibility Testing. The initial bacterial concentration was measured by a microsample spectrophotometer (Nanodrop 2000). The number of bacteria was determined by colony-forming unit (CFU) using the Miles and Misra method (i.e., colony counting) ${ }^{30}$ Prior to the experiment, E. coli samples were inoculated in Mueller Hinton (MH) broth, grown to optical density (OD600) to approximately 0.2 , and diluted with $\mathrm{MH}$ broth with or without antibiotic. The antibiotics and media were prewarmed at $37^{\circ} \mathrm{C}$ for $20 \mathrm{~min}$. The antibiotics were mixed with the bacteria before being injected into microchannels. The final concentration of the antibiotics are AMP $32 \mu \mathrm{g} / \mathrm{mL}, \mathrm{CIP} 4 \mu \mathrm{g} / \mathrm{mL}$, and SXT $4 /$ $76 \mu \mathrm{g} / \mathrm{mL}$. For AST with pathogens in urine, the urine sample was premixed with Mueller-Hinton media at 1:1 ratio. This protocol has been successfully demonstrated in our previous study. ${ }^{23}$ The single cell AST chip was placed on the microscope heating stage and preheated at $37{ }^{\circ} \mathrm{C}$ for $5 \mathrm{~min}$, and then, the sample was loaded into the channel. The growth rate, $\mu$, is estimated by

$$
\mu=\frac{\ln \frac{L\left(t_{0}+\Delta t\right)}{L\left(t_{0}\right)}}{\Delta t}
$$

where $L(t)$ denotes the length of interest. $\Delta t$ and $t_{0}$ denote the assay time and initial time, respectively. In our experiment, the spacing between bacteria is generally uniform and we do not observe issues of using the length for measuring the bacterial growth rate. If necessary, the gap distance between bacteria can be determined from the image and subtracted in the estimation of the growth rate. To evaluate the effectiveness of electrokinetic loading, we estimate the trapping rate to compare the efficiency at different voltages. Trapping rates are defined by the percentage of the channel that has bacteria trapped at the desired locations inside the channel. Data are reported as mean \pm standard deviation.

\section{RESULTS AND DISCUSSION}

Microfluidic Confinement of Individual Bacteria. The effects of the channel width on bacteria loading and confinement were first evaluated. The bacterial samples were loaded into confinement channels from $500 \mathrm{~nm}$ to $10 \mu \mathrm{m}$ by capillary force. For confinement channels with a small width $(\leq 1 \mu \mathrm{m})$, most bacteria were blocked at the entrance of the channel and only a small portion of bacteria could be loaded into the confinement channel. For large channels $(\geq 3 \mu \mathrm{m})$, bacteria were loaded into the channel; however, most bacteria were not confined, which could introduce uncertainty in the growth rate measurement. Therefore, a $2 \mu \mathrm{m}$ channel, which is optimized for both loading and confinement, was chosen for the single cell AST experiment. A typical E. coli loading experiment with $2 \mu \mathrm{m}$ channels is shown in Figure 1c. Bacteria were loaded into different locations of the channels. Due to the small size of the confinement channel, the bacteria were physically trapped and the growth of the bacteria could be monitored continuously. Figure $1 \mathrm{~d}$ shows time-lapse images of bacteria growing inside the confinement channels. The bacteria were observed to grow in length and divide into two daughter cells, and the daughter cells continuing to grow and divide. The total length increased exponentially and the doubling time of the bacteria could be extracted from the images. For the E. coli clinical isolates used in this experiment, the average doubling time was determined to be $\sim 36 \mathrm{~min}$. This value is in reasonable agreement with the doubling time of typical pathogenic E. coli, which is between 20 and $60 \mathrm{~min} .{ }^{31}$ These results demonstrate the applicability of confinement microchannels for monitoring the bacterial growth at the single cell level.

Electrokinetics-Assisted Pathogen Loading. In the microfluidic confinement experiment, the location and the number of bacteria in the channels are generally random and are difficult to control for AST. To facilitate the active loading of bacteria into defined locations inside the channel, electrokinetic loading is applied via microelectrodes integrated perpendicularly to the channel. ${ }^{32,33}$ Figure 2 a illustrates the

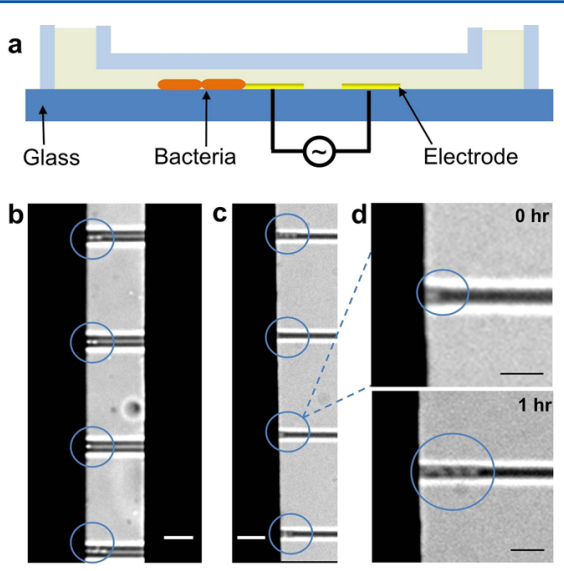

Figure 2. (a) A schematic diagram (cross-section view) of a pair of parallel electrodes with the bacteria positioned by DEP force and trapped in the microchannel. (b,c) E. coli trapped by AC electrokinetic force. E. coli bacteria were trapped at (b) the inner edge and (c) the outer edge. (d) Time lapse images of the bacterium in (c) with $1 \mathrm{~h}$ inoculation. Scale bars are $10 \mu \mathrm{m}$.

design of the device. The ability to position bacteria could also simplify and automate the observation procedure, since multiple bacteria can be located in defined locations in the same field of view. In our design, microelectrodes are aligned near the inlet $(\sim 400 \mu \mathrm{m})$ to reduce the probability that a bacterium is physically trapped in the channel before reaching the electrode region. It should be noted that DEP is typically operated in low conductivity buffers to maximize the effective polarization and to avoid electrolysis. ${ }^{34-36}$ We have overcome these issues by using a $\mathrm{Ti}-\mathrm{Au}-\mathrm{Ti}$ sandwich electrode configuration, which has high stability for electrokinetic operation and can endure high voltages for an extended period of time without observable degradation. ${ }^{37}$ This configuration allows us to manipulate $E$. coli in standard high conductivity AST media ( $\mathrm{MH}$ broth) without media dilution or replacement. Furthermore, the bacteria are in close proximity with the electrode edge; therefore, only a small applied voltage is required to trap the bacteria.

In the electrokinetic loading experiment, the sample solution is pipetted to the entrance of the microchannels. The capillary 
driven fluid motion entrains the bacteria to regions near the electrode edge, and the bacteria are trapped on the edge by DEP. In the experiment (Figure 2b,c), we applied an AC potential of $5 \mathrm{~V}$ peak to peak at $1 \mathrm{MHz}$. E. coli bacteria were observed to experience positive DEP and were trapped at the edges of the electrode. The bacteria can be trapped at both the outer edges and inner edges between the electrodes (Figure $2 \mathrm{~b}, \mathrm{c})$. For a larger channel such as a $6 \mu \mathrm{m}$ channel (Figure $2 \mathrm{~b}$ ), bacteria can be trapped at the inner edges. However, for a $2 \mu \mathrm{m}$ channel (Figure $2 c, d$ ), bacteria were generally trapped at the outer edges before they reach the inner edges. Since the bacteria reach the outer edge first, we optimize the operating parameters for trapping bacteria at the outer edges. Upon loading, the electric field can be removed and the bacteria remain confined in the $2 \mu \mathrm{m}$ channels. On the electrode edge, the bacteria grew normally with a growth rate similar to the condition without electrokinetic loading (Figure 2d). To optimize the loading efficiency, which is the percentage of channels loaded with one or more bacteria, the applied voltage was adjusted from 0 to $8 \mathrm{~V}$ systematically (Figure $3 \mathrm{a}$ ). Without
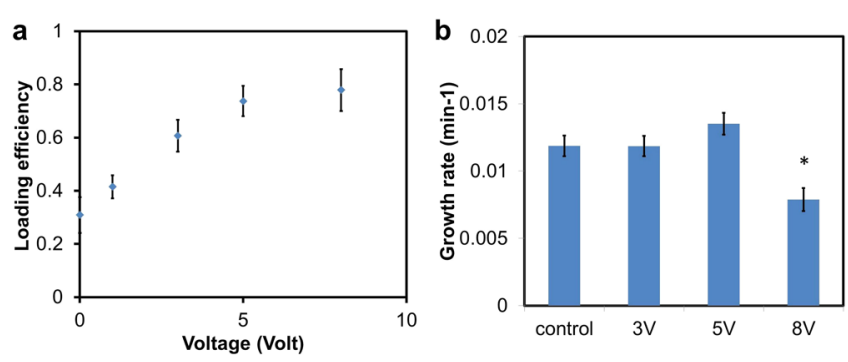

Figure 3. Electrokinetic loading of bacteria. (a) Dependence of the loading efficiency on the applied voltage. (b) Dependence of the growth rate on the applied voltage. $(* p<0.05)$. Data represent mean \pm standard error.

an external electric potential (i.e., $0 \mathrm{~V}$ ), the loading efficiency is slightly above $30 \%$. The high value is likely due to physical blockage by the electrode, which has a height of $250 \mathrm{~nm}$. The loading efficiency generally increases with the voltage from 0 to $5 \mathrm{~V}$ and reaches a plateau of $\sim 75 \%$ at $5 \mathrm{~V}$.

A high applied voltage could potentially introduce unwanted effects that affect the bacterial growth rate. The effect of the applied voltage on the bacterial growth rate is therefore characterized (Figure 3b). In the experiment, the bacteria growth rates are not affected at or below $5 \mathrm{~V}$. With a higher voltage (e.g., $8 \mathrm{~V}$ ), a reduction of the bacterial growth rate was observed. The reduction in growth rate could be explained by the heat generated (Joule heating) during electrokinetic operation. The importance of the electrothermal effect is supported by the fact that the proper operating voltage range can be affected by the temperature of the heating stage. For parallel electrodes, the maximum temperature rise due to electrokinetic operation can be approximated by $\Delta T=\sigma V_{\mathrm{rms}}^{2} /$ $(8 k) .^{38}$ For water (thermal conductivity $k=0.6 \mathrm{~J} /(\mathrm{msK})$ and electrical conductivity $\sigma=1.3 \mathrm{~S} / \mathrm{m}$ ) with an applied voltage $V_{\text {rms }}=8 \mathrm{~V}$, the temperature rise is estimated to be $\Delta T=17.3$ ${ }^{\circ} \mathrm{C}$ resulting in a maximum temperature of $54{ }^{\circ} \mathrm{C}$. The high temperature could reduce the bacterial growth rate. ${ }^{39}$ For $5 \mathrm{~V}$, the maximum temperature rise is approximately $6.8{ }^{\circ} \mathrm{C}$. It should also be noted that the temperature experienced by the bacteria is lower than the maximum temperature calculated since the electrode is serving as a thermal heat sink and creates a local temperature distribution. In our experiment, $5 \mathrm{~V}$ is able to achieve over $75 \%$ loading efficiency without affecting the bacterial growth rate and is, therefore, optimized for the single AST experiment.

Kinetics and Distribution of Individual Bacterial Growth Rates in Confined Channels. The kinetics and the distribution of the bacterial growth were then evaluated at the single bacterium level. The information will be useful to understand the growth behavior of individual bacteria and to determine the optimal conditions for single cell AST for statistical analysis. Furthermore, little is known on the individual response of bacteria with antibiotic below the minimum inhibitory concentration. Figure 4a illustrates typical
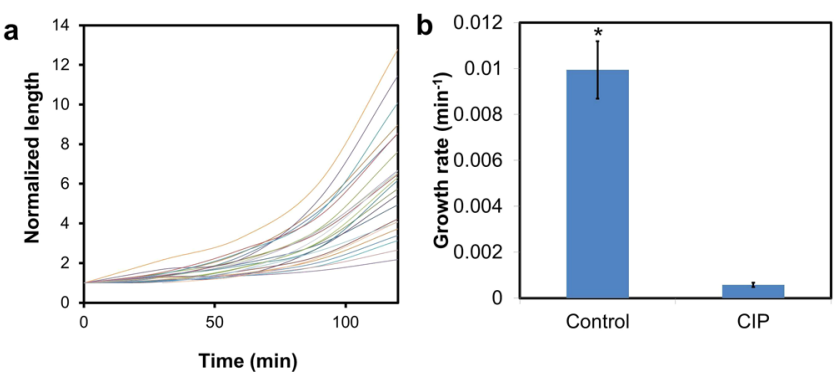

Figure 4. Single cell AST. (a) Growth of individual E. coli in confined microchannels. (b) Bacterial growth rates with and without antibiotic $\left({ }^{*} p<0.01 ; \mathrm{CIP}=\right.$ ciprofloxacin $)$.

growth curves of individual bacteria in $\mathrm{MH}$ broth without antibiotic. The length of the bacteria increases exponentially in the confined channel. We observed a large variation of growth rates among the bacteria; nevertheless, the data can generally be fitted by exponential curves. The growth could be easily observed with bright field or phase contrast microscopy. With the application of an antibiotic (e.g., ciprofloxacin), the bacteria did not display an observable growth (data not shown). As a result, the bacterial growth rates with and without antibiotic can be clearly distinguished in as short as $30 \mathrm{~min}$ (Figure 4b). The bacterial growth rate is found to be a more reliable parameter for judging bacterial growth than the absolute length due to uncertainty in optical resolution. These results indicate that phenotypic AST with confinement microchannels and optical microscopy can be finished with a time comparable to the doubling time of the bacteria.

The distribution of the bacterial growth rate among the population is further investigated. Figure 5a shows the histograms of the $E$. coli growth rate under different concentrations of ciprofloxacin. Without antibiotic, the bacterial growth rates show a Gaussian distribution and have an average

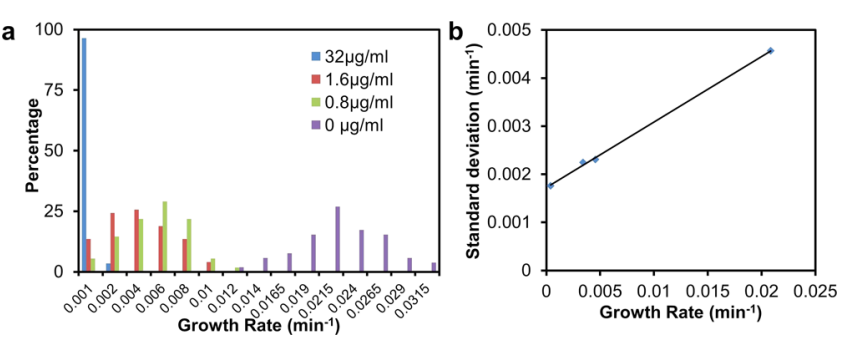

Figure 5. (a) Distribution of growth rates of EC137 with different ciprofloxacin concentrations. (b) Relationship between the mean and stnadard deviation of the bacterial growth rate. 

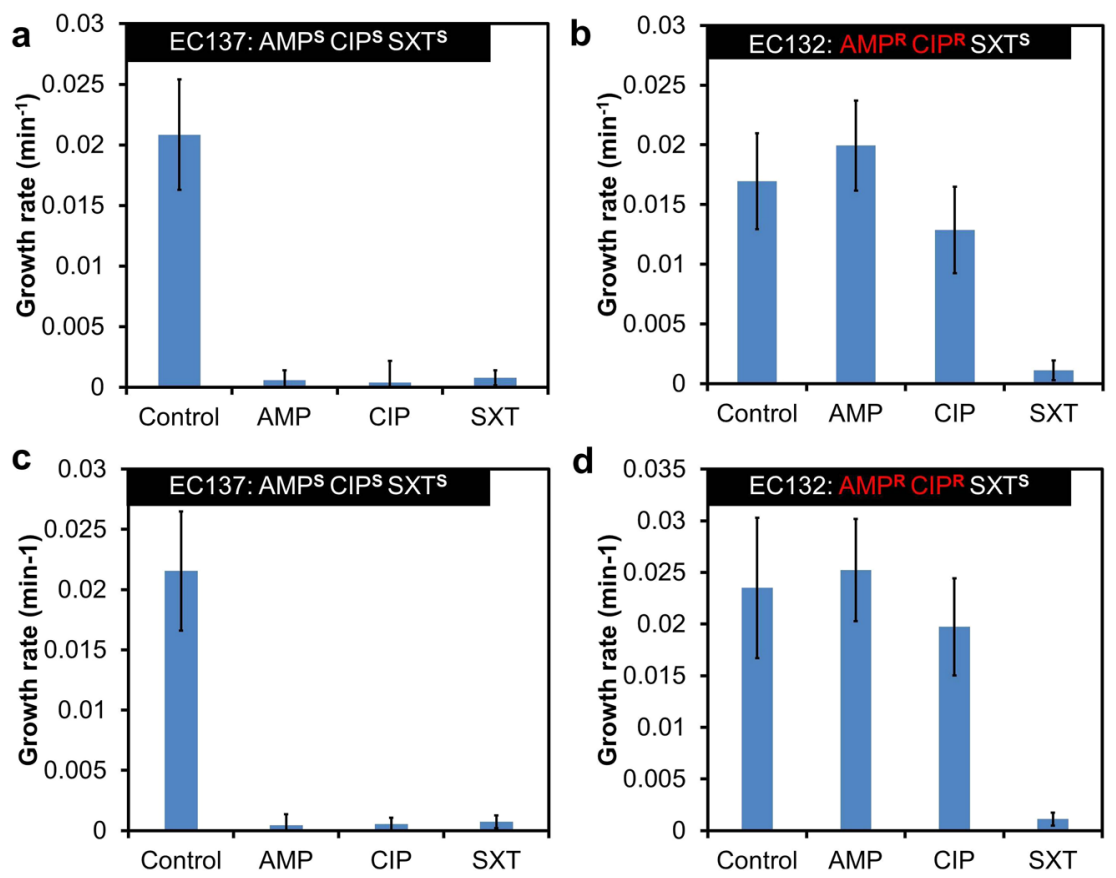

Figure 6. Single cell AST for antibiotic resistance profiling. (a, b) Uropathogenic clinical isolates EC137 and EC132 in Mueller-Hinton media were tested with different antibiotics. (c, d) The clinical isolates in urine were tested with different antibiotics. The urine samples were mixed with Mueller-Hinton media at a 1:1 ratio. Ampicillin (AMP); ciprofloxacin (CIP); trimethoprim/sulfamethoxazole (SXT). The superscripts "S" and "R" refer to sensitive and resistant to the antibiotics.

value of $\sim 0.02 \mathrm{~min}^{-1}$. At a ciprofloxacin concentration below the minimum inhibitory concentration (e.g., 0.8 or $1.6 \mu \mathrm{g} / \mathrm{mL}$ ), the distribution remains Gaussian and the mean growth rate decreases as the antibiotic concentration increases (Figure 5a). Total inhibition of the bacterial growth was observed at $32 \mu \mathrm{g} /$ $\mathrm{mL}{ }^{40}$ The variation in the growth rate generally increases linearly as the growth rate increases (Figure $5 b$ ). Interestingly, our results also suggest that the distribution shifts smoothly with the drug concentration indicating an analog response of the antimicrobial effect on the bacterial growth rate. The mechanistic reason of why the distribution shifts in a continuous manner could be understood by the mechanism of action of ciprofloxacin, which inhibits DNA gyrase and stops division. ${ }^{41}$ Therefore, a small amount of ciprofloxacin could partially slow down the growth rate.

Antimicrobial Resistance Profiling of Uropathogenic Clinical Isolates. The confinement channel was then applied for single cell AST. We chose two uropathogenic E. coli clinical isolates (EC132 and EC137) with four different conditions (control, AMP, CIP, and SXT) to evaluate the applicability of confined microchannels for rapid antibiotic resistance profiling. The assay time was chosen to be $1 \mathrm{~h}$. From clinical microbiology analysis, ${ }^{13}$ it was previously determined EC137 is sensitive to all three antibiotics, and EC132 is resistant to ampicillin and ciprofloxacin but not trimethoprim/sulfamethoxazole. The growth rate without antibiotics for the EC137 strain is observed to be around $0.02 \mathrm{~min}^{-1}$, which is at least 25 times higher than the conditions with antibiotics (Figure 6a). Compared to the control, EC132 shows a comparable growth rate with ampicillin and ciprofloxacin and displays a significantly lower value with trimethoprim/sulfamethoxazole (Figure 6b). In order to explore the feasibility and applicability of single cell AST for physiological fluids, the AST experiments were performed with uropathogens spiked in urine (Figure $6 c, d)$. Interestingly, the uropathogen EC 132 has a higher growth rate when cultured in urine, as also observed in our previous study. ${ }^{23}$ Nevertheless, the AST results and time required are consistent with the data in $\mathrm{MH}$ broth only and in the clinical microbiology laboratory. These results suggest that antibiotic resistance profiling can be finished in $1 \mathrm{~h}$ using the single cell AST approach.

In this study, we demonstrate confinement microchannels for single cell AST. AC electrokinetics is also shown to enhance the loading rate of bacteria to desired locations inside the channel. ${ }^{34}$ While most electrokinetic techniques only function effectively in low conductivity DEP buffers, our device allows effective operation in high conductivity samples, such as AST buffers $(\sim 1 \mathrm{~S} / \mathrm{m})$ that is required for AST. These results demonstrate an effective approach for rapid AST. The major advantages of the device are that single cell AST can be finished in less than $1 \mathrm{~h}$ and that the device requires only a small sample volume $(\sim 5 \mu \mathrm{L})$ for each test. Compared to previous works using stochastic confinement with fluorescence indicators, ${ }^{25}$ the microfluidic confinement approach allows direct observation of the bacterial growth with standard bright field microscopy, which reduces the total assay time. Furthermore, on-chip monitoring of the activity of individual bacteria may provide a useful technique to investigate the bacteria-antibiotic interaction at the single cell level. In addition, the microfluidic platform can be easily integrated with other detection systems or postanalysis systems in the future. For example, impedimetric sensing could potentially be incorporated into the system for quantifying the growth rate without optical microscopy. ${ }^{42}$ Alternatively, cell-phone-based detection systems with image analysis algorithms could also be applied to automate the process, which may facilitate the implementation in resource-limited settings. 


\section{CONCLUSION}

This paper has demonstrated a microfluidic device for the culture of individual bacteria and rapid AST using confined microchannels and electrokinetic loading. The promising results will form the foundation for using confined microchannels and $\mathrm{AC}$ electrokinetics for addressing the technical challenges in rapid AST at the point of care. With further development, we envision that single cell AST will be adopted in various situations for clinical management of infectious diseases.

\section{AUTHOR INFORMATION}

\section{Corresponding Author}

*Tel: +1-520-626-2215. Fax: +1 520-621-8191. E-mail: pak@ email.arizona.edu.

\section{Notes}

The authors declare no competing financial interest.

\section{ACKNOWLEDGMENTS}

We thank ASU Center for Solid State Electronics Research for technical assistance in fabrication of microchannels. The authors thank Lindsay Bahureksa and Ariana Lamanda for technical support and graphic preparation. This work was supported by NIH Health Director's New Innovator Award (1DP2OD007161-01) and NIAID (1U01AI082457-01 and 2R44AI088756-03).

\section{REFERENCES}

(1) Pinner, R. W.; Teutsch, S. M.; Simonsen, L.; Klug, L. A.; Graber, J. M.; Clarke, M. J.; Berkelman, R. L. JAMA, J. Am. Med. Assoc. 1996, 275, 189-193.

(2) Hawkey, P. M. J. Antimicrob. Chemother. 2008, 62, I1-I9.

(3) Daum, R. S.; Ito, T.; Hiramatsu, K.; Hussain, F.; Mongkolrattanothai, K.; Jamklang, M.; Boyle-Wang, S. J. Infect. Dis. 2002, 186, 1344-1347.

(4) Herold, B. C.; Immergluck, L. C.; Maranan, M. C.; Lauderdale, D. S.; Gaskin, R. E.; Boyle-Vavra, S.; Leitch, C. D.; Daum, R. S. JAMA, J. Am. Med. Assoc. 1998, 279, 593-598.

(5) Huttner, B.; Harbarth, S. PLoS Med. 2009, 6, No. e1000080.

(6) Kuper, K. M.; Boles, D. M.; Mohr, J. E.; Wanger, A. Pharmacotherapy 2009, 29, 1326-1343.

(7) Pfaller, M. A.; Jones, R. N. Arch. Pathol. Lab. Med. 2006, 130, 767-778.

(8) Levy, S. B.; Marshall, B. Nat. Med. 2004, 10, S122-S129.

(9) Mach, K. E.; Wong, P. K.; Liao, J. C. Trends Pharmacol. Sci. 2011, $32,330-336$.

(10) Chen, C. H.; Gau, V.; Zhang, D. D.; Liao, J. C.; Wang, F.-Y.; Wong, P. K. PloS One 2010, 5, No. e15472.

(11) Kim, M.; Yoda, M. Exp. Fluids 2010, 49, 257-266.

(12) Daniels, J. S.; Pourmand, N. Electroanalysis 2007, 19, 12391257.

(13) Mach, K. E.; Mohan, R.; Baron, E. J.; Shih, M. C.; Gau, V.; Wong, P. K.; Liao, J. C. J. Urol. 2011, 185, 148-153.

(14) Murray, P. R.; Niles, A. C.; Heeren, R. L. J. Clin. Microbiol. 1987, $25,2372-2377$.

(15) Ljungholm, K.; Wadso, I.; Mardh, P. A. J. Gen. Microbiol. 1976, 96, 283-288.

(16) Deblanc, H. J.; Wagner, H. N.; Charache, P. Antimicrob. Agents Chemother. 1972, 22, 360.

(17) Isenberg, H. D.; Maclowry, J. D. Annu. Rev. Microbiol. 1976, 30, 483-505.

(18) Gfeller, K. Y.; Nugaeva, N.; Hegner, M. Biosens. Bioelectron. 2005, 21, 528-533.

(19) Ertl, P.; Robello, E.; Battaglini, F.; Mikkelsen, S. R. Anal. Chem. 2000, 72, 4957-4964.
(20) Mann, T. S.; Mikkelsen, S. R. Anal. Chem. 2008, 80, 843-848.

(21) Sin, M. L.; Gao, J.; Liao, J. C.; Wong, P. K. J. Biol. Eng. 2011, 5, 6.

(22) Wang, T. H.; Wong, P. K. J. Assoc. Lab. Autom. 2010, 15, A15A16.

(23) Chen, C. H.; Lu, Y.; Sin, M. L. Y.; Mach, K. E.; Zhang, D. D.; Gau, V.; Liao, J. C.; Wong, P. K. Anal. Chem. 2010, 82, 1012-1019.

(24) Peitz, I.; van Leeuwen, R. Lab Chip 2010, 10, 2944-2951.

(25) Yin, H. B.; Marshall, D. Curr. Opin. Biotechnol. 2012, 23, 110119.

(26) Boedicker, J. Q.; Li, L.; Kline, T. R.; Ismagilov, R. F. Lab Chip 2008, 8, 1265-1272.

(27) Long, Z.; Nugent, E.; Javer, A.; Cicuta, P.; Sclavi, B.; Cosentino Lagomarsino, M.; Dorfman, K. D. Lab Chip 2013, 13, 947-954.

(28) Moffitt, J. R.; Lee, J. B.; Cluzel, P. Lab Chip 2012, 12, 14871494.

(29) Balaban, N. Q.; Merrin, J.; Chait, R.; Kowalik, L.; Leibler, S. Science 2004, 305, 1622-1625.

(30) Miles, A. A. Cc/Life Sci. 1979, L12-L12.

(31) Bernstein, J. A.; Khodursky, A. B.; Lin, P. H.; Lin-Chao, S.; Cohen, S. N. Proc. Natl. Acad. Sci. U.S.A. 2002, 99, 9697-9702.

(32) Wong, P. K.; Wang, T. H.; Deval, J. H.; Ho, C. M. IEEE-ASME Trans. Mechatronics 2004, 9, 366-376.

(33) Castellanos, A.; Ramos, A.; Gonzalez, A.; Green, N. G.; Morgan, H. J. Phys. D: Appl. Phys. 2003, 36, 2584-2597.

(34) Sin, M. L. Y.; Shimabukuro, Y.; Wong, P. K. Nanotechnology 2009, 20, 165701.

(35) Sin, M. L. Y.; Gau, V.; Liao, J. C.; Haake, D. A.; Wong, P. K. J. Phys. Chem. C 2009, 113, 6561-6565.

(36) Wong, P. K.; Chen, C. Y.; Wang, T. H.; Ho, C. M. Anal. Chem. 2004, 76, 6908-6914.

(37) Gao, J.; Sin, M. L. Y.; Liu, T. T.; Gau, V.; Liao, J. C.; Wong, P. K. Lab Chip 2011, 11, 1770-1775.

(38) Ramos, A.; Morgan, H.; Green, N. G.; Castellanos, A. J. Phys. D: Appl. Phys. 1998, 31, 2338-2353.

(39) Ron, E. Z.; Davis, B. D. J. Bacteriol. 1971, 107, 391.

(40) Andrews, J. M. J. Antimicrob. Chemother. 2001, 48, 5-16.

(41) Drlica, K.; Zhao, X. L. Microbiol. Mol. Biol. Rev. 1997, 61, 377.

(42) Silley, P.; Forsythe, S. J. Appl. Bacteriol. 1996, 80, 233-243. 